\title{
Extended Taxonomy of Rule Extraction Techniques and Assessment of KDRuleEx
}

\author{
Kamal Kumar Sethi \\ Professor \& Head, IT \\ Acropolis (AITR), Indore, \\ Indore, M.P., India
}

\author{
Durgesh Kumar Mishra \\ Professor \& Head, CSE \\ Sri Aurobindo (SAIT), Indore, \\ Indore, M.P., India
}

\author{
Bharat Mishra \\ Reader \\ MGCGV University, Chitrakoot, \\ Chitrakoot, M.P., India
}

\section{"If you cannot measure it, you cannot improve it." - William Kelvin}

\begin{abstract}
Classifiers like ANN \& SVM are always preferred over other classification model like decision tree due to higher accuracy but lacking explainability and comprehensibility. Rule extraction techniques bridges gap between accuracy and comprehensibility. To evaluate and compare different rule extraction techniques, we require measures for evaluation and categorization. Taxonomy helps us to select a technique based on the requirements and desired priorities. In this paper, we extended popular ADT-taxonomy of rule extraction which has been designed for ANN as underlying model. Proposed taxonomy covers all types of work related to rule extraction. It makes easier to introduce new rule extraction techniques by improving the performance on evaluation criteria. In this paper almost all possible aspects of evaluation and categorization of rule extraction techniques has been considered and further used to evaluate the algorithm KDRuleEx.
\end{abstract}

\section{General Terms}

Data Mining, Rule Extraction, Decision Systems.

\section{Keywords}

Rule Extraction, Taxonomy, Decision Table, Accuracy, Fidelity, Comprehensibility, Consistency, Translucency.

\section{INTRODUCTION}

Rule extraction technique has great importance in predictive data mining and machine learning research area. Rule extraction techniques can represent extracted model in form of If-Then rules, $\mathrm{M}$ of $\mathrm{N}$ rules, decision tree, decision table, etc. Opaque models like ANN and SVM shows better accuracy performance in comparison to white box or transparent model like decision tree [1][2][3]. Rule extraction is the task of making an opaque model transparent and hopefully comprehensible [4]. Comprehensibility is necessary in many application areas and to make neural networks more comprehensible we need rule extraction from neural network. Two decades have been past from the inception of rule extraction techniques, many researchers have proposed and developed new models and algorithms for rule extraction. Now it is very difficult to select one best amongst them for a specific domain due to less work on the categorization and comparative studies of these techniques.

Diederich argued for the application of assessment and evaluation techniques [5]. It motivates us to review available work on taxonomy for rule extraction techniques and modified them to include all aspects of the evaluation. Further we evaluated KDRuleEx technique to assess the efficacy of revised taxonomy and position of KDRuleEx. For some domains accuracy is sufficient but domains related to medicine, safety, etc, comprehensibility as well as explainability is equally important[6]. The explainability of rules using decision table is better than other ways of representation. Decision tables are widely used in knowledge representation system. Using decision table user can understand the rules easily in comparison to decision tree [7][8][9]. The work for KDRuleEx algorithm has been motivated from the fact that a classifier should have accuracy, comprehensibility and explainability[10].

In this paper, many criteria are identified for assessing rule extraction techniques and a new taxonomy based on these criteria is proposed. Performance and categorization on each of these categories are rated on ordinal scale, to summarize their relative strengths and weaknesses. This information will be useful for analysts to select best rule extraction technique to use, as well as to researchers to propose improved techniques. Further we summarized applicability report, details of the evaluation and the scoring of KDRuleEx on all criteria of proposed taxonomy. We have also discussed important research areas in rule extraction.

\section{LITERATURE SURVEY}

Many researchers have discussed rule extraction techniques, their evaluation criterion and taxonomy [11], [12], [13], [14]. Most of the researchers have included few aspects in their discussion [6], [15]. A new taxonomy for rule extraction techniques can be devised by including all evaluation criteria from previously discussed taxonomies. Rule extraction technique must be designed in such a manner so that performance of algorithm must be optimum on all aspects of taxonomy. Large numbers of rule extraction techniques are available but their performance is acceptable only on few evaluation criterion and the scope of improvement is there. In KD taxonomy, we have integrated different aspects proposed by various researchers. In most of the situation, to adopt or propose a suitable rule extraction technique is not an easy job because there are many influencing factors to be considered. Duch has speculated that most research papers on the rule extraction are usually limited to the description of new algorithms and only partial solutions to the problem of rule extraction [16]. Problem areas like tradeoff between comprehensibility, fidelity and accuracy, optimization of the linguistic variables and final rules, estimation of the consistency, coverage and reliability of rules generally ignored. To answer these problems one may start from a less accurate simple description of the data and then can transform it into more accurate complex description rules or may start with accurate opaque model and can transform into more comprehensible description [36] [37]. In development of KDRuleEx, all necessary aspects of taxonomy and evaluation measures have been considered. 
Andrews et al[6] and Tickle et al [17] have suggested criterions for the classification of rule extraction techniques. They have incorporated five properties of rule extraction to categorize rule extraction techniques. These are-

Expressive power of the extracted rules describe the form of the extracted rules.

Quality of the extracted rules may be measured using accuracy, fidelity, consistency, comprehensibility.

Translucency is the view taken within the rule extraction technique of the underlying Artificial Neural Network units. It can be evaluated as decompositional, pedagogical and eclectic depending upon the degrees of granularity.

Algorithmic complexity of rule extraction technique.

Portability is the rule extraction technique across various ANN architectures. It describes extent to which the underlying ANN incorporates specialized training regimes.

Huysmans et al has also given the taxonomy of rule extraction algorithm on broader perspective [15] by suggesting 3 points classification of rule extraction techniques-

Scope: The type of problem covered by technique; may be classification or regression.

Type of dependency on underlying model: may be categorized as independent or dependent. If it is independent then same algorithm can be used with different type of underlying model.

Format of extracted model: may be categorized predictive or descriptive. A technique called predictive if it is exclusive and exhaustively cover whole population else descriptive.

\section{KD TAXONOMY OF RULE EXTRACTION}

In broader sense, few points discussed by Huysmans [15] are similar to ADT taxonomy [6]. But points discussed in ADT taxonomy mainly cover techniques of Rule Extraction from ANN whereas Huysmans has taken all types of underlying model in account. After reviewing the above discussed taxonomy and work of others researches, we can conclude that there is need of assessment criterion set with inherent characteristics of generality and specialty to cover all the aspects of assessment. This motivated us to propose new KD taxonomy for rule extraction techniques. To study and evaluate rule extraction techniques we need to answer following questions:

1. What kind of data set is to be examined? \{Input Domain\}

2. What kind of model is to be used as an opaque model? \{Underlying Model $\}$

3. What rule extraction paradigm would be best to use? \{Techniques\}

4. How do we represent extracted knowledge / model? \{Representation of Extracted Model $\}$

5. How do we measure performance of extracted model?\{Accuracy/ Fidelity/ Comprehensibility/ Efficiency\}

6. Whether extracted model is consistent or not? \{Algorithm/ evaluation criteria to measure consistency\}

7. How do we test the quality of extracted model? \{Coverage/ Comprehensibility/ Consistency/ Rule Quality

8. For what kind of problem the extracted model can be used? \{Classification / Regression\}

Based on questions raised, we have identified following measures for taxonomy of rule extraction techniques.

Input domain: Input domain refers to different types of data values used in data set. Input domain may be continuous (numeric value), categorical (non numeric value), binary and ordinal (order of values has importance). Most of the available algorithms are capable to work with specific type of input domain.

Underlying model: Rule extraction is the process of transforming accurate opaque model in to reasonably accurate comprehensible transparent model. ANN and the SVM are the most accurate known classification model which lacks on comprehensibility ground. Most of the work in rule extraction has been done from these two underlying model but the choices are not limited to these two.

Extracted model: Output model extracted using rule extraction technique need not to be in form of rules only. Many formats are used to represent the extracted model including if then rules, oblique rules, equation rules, boolean notation, propositional logic, first order logic, decision tree, decision diagram, finite automata, fuzzy logic, decision table, scientific law, etc [17]. Provision to convert from one type of representation to another will add an added advantage to model.

Scope: Predictive classifier model is basically used to predict the class label of continuous or categorical values. Predicting the class label for categorical values is known as classification while for continuous values is known as regression. Depending upon type of problem answered, scope of rule extraction technique may be classification or regression.

Dependency: Whether algorithm is dependent upon the type of underlying model i.e. ANN or SVM. An algorithm may be treated as independent if choice of model (ANN/ SVM) does not affect the working of rule extraction and it extracts rules from all kind of input model.

Translucency: Another way of classifying rule extraction algorithms is based on how the rule extraction algorithms monitor the trained neural net. Rule extraction techniques can be categorized based on granularity level of underlying model (generally used with ANN). The use of ANN within rule extraction techniques can be explicit or implicit. Depending upon the degree of granularity of underlying ANN rule extraction techniques can be categorized in to decompositional, pedagogical and eclectic. If rule extraction techniques view maximum level of granularity of underlying model then such techniques comes under the categories of decompositional. An algorithm is said decompositional if rules are extracted at individual unit level and within the ANN solution and then these rules are used to form global relationships. Two popular decompositional rule extraction algorithms are N OF M and SUBSET [18]. If rule extraction techniques view minimum level of granularity of underlying model then such techniques comes under the categories of pedagogical. In pedagogical technique, the ANN or underlying model is treated as a "black box" and extracted global relationship from direct mapping between input and output, without analyzing the detailed characteristic of underlying ANN model. Pedagogical rule extraction technique refers to modeling the function from input variables to predictions from the opaque model. Two popular examples of pedagogical rule extraction algorithms are TREPAN[19] and ANN-DT[20]. If Rule Extraction techniques incorporate elements of both pedagogical and decompositional then such techniques known as eclectic techniques [35] and DEDEC is popular example of this kind [21].

Portability: Portability is based on their dependency on the structure of the underlying neural network. A portable rule extraction technique may run on multiple type of input models varying in architecture of ANN. The architecture includes topologies, activation transfer functions, input and output encodings and training methods, type of ANN, number of layer and neurons, etc. The portability describes how well a rule extraction technique covers different type of ANN 
architecture available. It gives an idea about the extent to which the method requires special training regimes or placement of restrictions on network architectures. On the basis of portability an algorithm may be classified as general and specific. A portable technique seems to be more attractive because it may be used with variety of network architecture.

Algorithmic Complexity: This concerns with how much time and space a rule extraction algorithm requires. An algorithm will have high complexity if it is exponential, low if it is linear and moderate if it has polynomial time complexity. The complexity of a rule extraction algorithm is a challenge [22]. Using heuristics, the actual time and space requirement can be controlled. Although complexity is a very important factor for the applicability of a technique to real time application but very few authors have discussed it in great detail.

Quality: Quality is one of the most important component of the taxonomy of RE. Rule extraction algorithms can be classified based on the excellence of the extracted rules to explain the knowledge embedded in the opaque model. Quality of the extracted rule can be evaluated on the basis of accuracy, fidelity, comprehensibility, consistency, coverage and rule quality which can be described by number of rules, length of the rule and coverage of the rule. Accuracy describes how much accurate predictions made for the unseen data from the extracted representations. Fidelity describes how much extracted representations mimic the model from where rules were extracted. Comprehensibility describes the extent to which extracted representations are comprehensible (size). Consistency describes all the instances has been classified same at different run. Coverage defines the percentage of population covered by a rule or model. If a extracted model is exclusive and exhaustive then it is known as predictive otherwise known as descriptive format [15]. A high quality classifier model has quality rules which are less in number with small rule length to cover all dataset instances.

Scalability: Rule extraction algorithm must be designed in such a way it can work efficiently with that large size data sets and underlying model. The scalability is very important criteria because non scalable rule extraction technique may not be use in real time applications. Rule extraction algorithm is called scalable if it is possible to apply it on the problems that are huge in some sense. Shavlik and Craven[11] defined scalability' as : "Scalability refers to how the running time of a rule extraction algorithm and the comprehensibility of its extracted models vary as a function of such factors as network, feature-set and training-set size." In his definition they have used running time and comprehensibility of the extracted model to define scalability. Use of comprehensibility to define scalability is important because the main objective of the rule extraction techniques to achieve comprehensibility.

\section{ASSESSMENT CRITERION FOR RULE EXTRACTION TECHNIQUES:}

There are several criteria used for evaluating rule extraction algorithms. Craven and Shavlik had used five criteria for evaluation of rule extraction techniques listed below [11]:

Accuracy: Accuracy is ability to generalize on unseen data and shows the extent to which the extracted model is able to classify a set of previously unseen examples from the problem domain correctly [6]. For regression problem it represents the ability of the extracted model to precisely predict the target variable for novel data so that there is low difference (error) between the predicted value and actual value. The accuracy is measured in percentage of instances classified correctly by extracted model.
Accuracy $=\frac{\text { Number of Instances Predicted Correctely }}{\text { Total Number of Instances }}$

Fidelity: Fidelity mimics the behavior of ANN. Fidelity shows the extent to which the extracted model mimics the behavior of the underlying model from which it is extracted. Fidelity is closely linked to the accuracy and it shows the extent to which extracted representations accurately model the underlying model from which they are extracted. It is the capability of the extracted representation to show that how well the derived representation imitates the performance of the underlying model. Zhou had argued that it may be hard to achieve high level of accuracy and fidelity at the same time, if the performance of underlying model is questionable [23]. The fidelity is the percentage of instances for which the predictions made by the underlying model and the extracted model are same.

Fidelity $=\frac{\text { No. of Instances Predicted Same as by Underlying Nodel }}{\text { Total Wumber of Instances }}$

Comprehensibility: Comprehensibility may be expressed using readability, size and number of rules. Comprehensibility elaborates the extent to which extracted representations are humanly understandable. The term comprehensibility is subjective and same model may be more or less comprehensible for different user depending upon their background. The term comprehensibility and transparency seems to be similar but are different; comprehensibility is related to size whereas transparency deals with working of model. But in general these two terms are used interchangeably in rule extraction area until not mentioned specifically. In this way, comprehensibility may be treated as the degree of easiness with which the user can understand the reasoning of system behind the predictions made. Comprehensibility gives the power of justification to the user and system. Comprehensibility is subjective in nature, but to evaluate a model on comprehensibility ground, generally number of rules and length of rule are taken into consideration. Domingos [24] had given justification for comprehensibility. He argued that if accuracy of two model is same than simpler model is better than other.

Consistency: Consistency describes similarities in rules from different Networks. Consistency is the degree to which generated model under different training sessions (underlying model and data choose for training) produce the same classifications of unseen instances [6]. Towell and Shavlik had used consistency for the assessment of rule extraction algorithms [18] and Johansson et al. defined consistency [25]: "An algorithm is consistent if it extracts similar rules every time it is applied to the same data set."

Rule extraction algorithm is consistent if the rules extracted from a specific model are similar between different runs. Consistency of extracted model is important because if the extracted model vary considerably between training sessions then it is difficult to scribe any priority to a particular model.

Efficiency: The efficiency of the algorithm can be measured in terms of execution time or required memory storage. This concerns with how much time and space a rule extraction algorithm requires. Using heuristics efficiency can be controlled and improved.

Coverage: Coverage or decision support degree can be defined as proportion of instances for which a classifier model makes a prediction. If a classifier does not classify all the 
instances then it would be important to know its performance on the set of instances for which it is confident to make prediction [26]. An extracted model is called complete or exhaustive if extracted model covers all the instances of the dataset. A model is called exclusive if no instance in dataset is covered by two rules.

Order of Rule: The order of rule is the number of conjunction is a boolean expression of the form

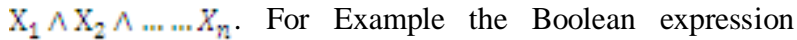
$\mathrm{X}_{1} \mathrm{AX}_{2} \wedge \mathrm{X}_{\mathrm{g}}$ is a rule of order 3 .

Different researchers have suggested different goals for rule extraction techniques and majority of them have argued for accuracy, fidelity, comprehensibility, efficiency of extracted model. The improvement of rule extraction algorithms is driven by these goals. Performance of the resulting rule extraction algorithm may depend on which of these goals is aimed at. It is desired that one and same algorithm should be generic enough to empower the user to choose his prioritized goal among these goals. Tradeoff between fidelity and comprehensibility, fidelity and accuracy, accuracy and comprehensibility [36], fidelity and computation time, etc should be answered through the setting of few parameters.

\section{RATIONALES FOR THE CHOICE MADE FOR UNDERLYING MODEL, EXTRACTED MODEL AND TRANSLUCENCY IN KDRULEEX}

In this section recently developed rule extraction algorithm KDRuleEx has been judged for the rationale for the choices made for underlying model, translucency and extracted model.

\subsection{ANN as Underlying Model:}

Generally rule extraction is done from opaque model like ANN and SVM. ANN and SVM are the most accurate known classifier and ANN gets a better result with higher accuracy, while the SVM classifier produces more noise and has some edge effects [28]. Keeping this in mind we have chosen ANN as underlying model for our study. Possible application of rule extraction is suggested by Andrews et al [6], Tickel et al [17] and Jacobsson [27] in the context of ANN and may be summarized as:

1. Provides explanation of the ANN: ANN is widely used in expert system and DSS. But knowledge representation and decision of ANN is not easily explainable. Rule extraction provides explanation capability to ANN which provides insight into the problem by understanding the internal architecture.

2. Acquires a generic model of the domain: In most of the cases due to generalization ability, ANN represents data better than data itself. But in case of insufficient data, this ability may get weak. A model with good interpretability improves power of generalization.

3. Allow verification/validation of the ANN: Rule extraction can be used to verify and validate the working of an ANN. As rule extraction provides user understandable model of underlying ANN, the working of ANN can be verified.

4. Improving current ANN architectures by identifying errors: Using rule extraction we can identify the errors or area of improvement in ANN architecture.

5. Use of ANN in safety critical problems: Enhancing the confidence while using ANN in safety critical problem domain like medical, diagnostic control by providing accurate decision which are justified also.

6. Better performance for unseen data by using ANN: Nonlinearity and generality and accuracy are the strength of
ANN. ANN is one of the most accurate classifier which has good performance on unseen dataset. Using rule extraction user may understand these unknown nonlinear relationships of dataset easily.

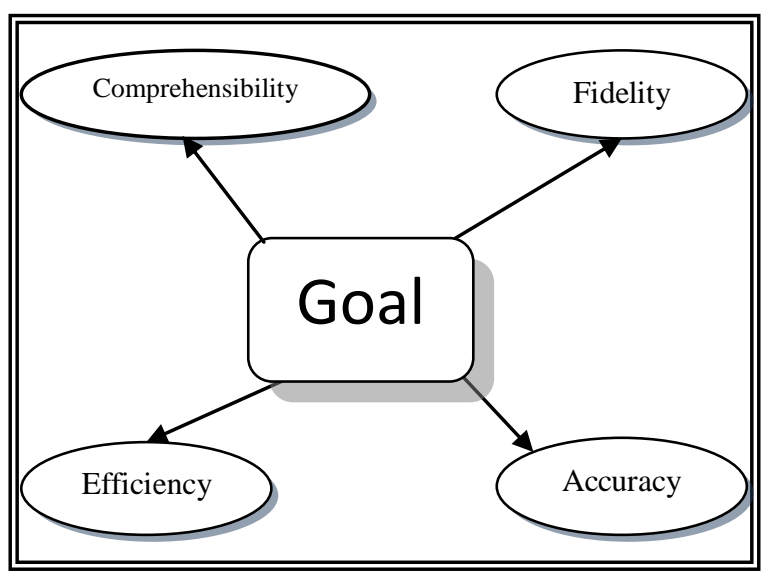

Figure 1: Four Possible Opposite Goals of Rule Extraction Techniques [27]

\subsection{Pedagogical model for Translucency:}

The main objective of rule extraction is dual, that is, extracted rules must satisfy both accuracy and comprehensibility requirement. The process of decompositional approach of rule extraction is tedious and results in complex and large descriptions. So the drawbacks of decompositional approach are time and computation limitations. Pedagogical approach may be faster than the decompositional, since they don't analyze the weights or internal architecture of the network, but they may somewhat less accurately capture rules describing the network's behavior. In addition, pedagogical approaches of rule extraction are architecturally independent. Pedagogical algorithms can be applied to variety of neural networks as it extracts rules by mapping the input output relationships. In general, the performance analysis shows that the pedagogical approach achieves better classification accuracy with higher rule comprehensibility over other technique. Pedagogical rule extraction techniques meet the generality criteria when rule extraction is done using ensembles and it is difficult to create a decompositional rule extraction technique that is general enough to work on an arbitrary ensemble[29]. In a nutshell, the pedagogical approaches are very effective and easy to use for extracting rules from ANN trained on large size datasets.

\subsection{Decision Table as Representation of Extracted Model:}

It is important that extracted knowledge should be correct, consistent, complete and non-redundant. The extracted model must be verified and validated and decision table provide assistance for extensive validation and verification [8]. It facilitates to check for contradictions, inconsistencies, incompleteness, redundancy in extracted model. Decision table are fastest, easiest, most confident and most accurate representation of a classifier [30]. Decision tree, oblique rule and propositional rule are slower, difficult and less confident. Decision tables provided a very useful support for expert systems construction and verification.

Decision tables frequently used in expert system, decision support system, knowledge representation, software engineering and artificial intelligence. Applications of decision table in real world domain are production systems, medicine system, and business rule representation etc. 
Decision table provides efficient representation of data and finds hidden patterns; offers straight forward interpretation of obtained results.

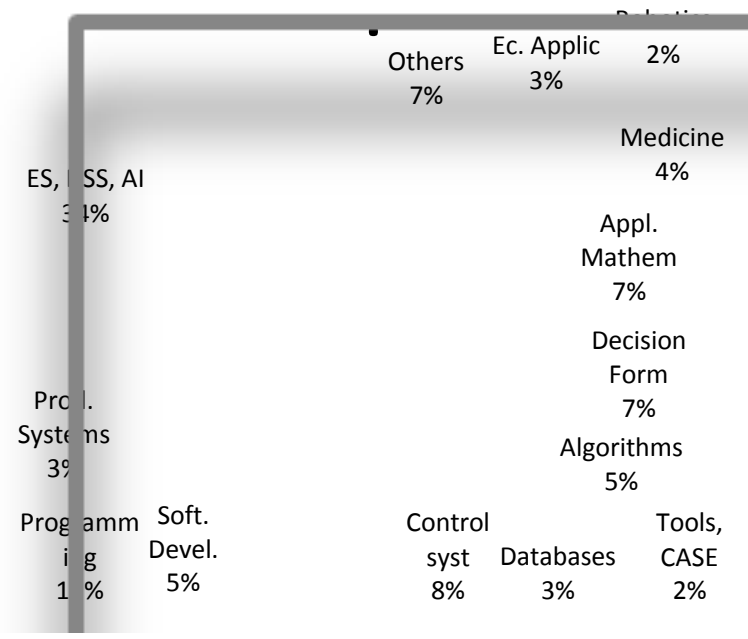

Figure 2: Use of Decision Table in Different Domains [31]

\section{ASSESSMENT OF KDRULEEX ALGORITHM ACCORDING TO KDTAXONOMY FOR RULE EXTRACTION TECHNIQUES}

KDRuleEx is a novel algorithm for Rule Extraction which extract transparent model in the form of decision table[10]. The rule extracted from the KDRuleEx are easily be transformed in to if then rule and decision tree. The algorithm is pedagogical and does not make any assumption for underlying model. The algorithm is independent upon the architecture of underlying model and portable in nature. The initial results were reported using ANN as underlying model but can easily be used with other underlying model too. KDRuleEx can be applied under almost any circumstances; it can deal with both categorical and numerical inputs and applicable for classification problems. The algorithm is non recursive in nature and hence shows superior performance on memory requirement. The algorithm shows high accuracy, fidelity and comprehensibility by adjusting the few parameters.

\begin{tabular}{|c|c|c|}
\hline $\begin{array}{c}\text { Evaluation } \\
\text { Parameter }\end{array}$ & $\begin{array}{c}\text { Universe of } \\
\text { discourse }\end{array}$ & $\begin{array}{c}\text { Evaluation of } \\
\text { KDRuleEx }\end{array}$ \\
\hline $\begin{array}{c}\text { Underlying } \\
\text { Model }\end{array}$ & ANN, SVM & $\begin{array}{c}\text { ANN\{can be used } \\
\text { with other models } \\
\text { also\} }\end{array}$ \\
\hline Output Model & $\begin{array}{c}\text { If-Then Rules, } \\
\text { Decision Tree, } \\
\text { Decision Table, } \\
\text { Decision Graph, } \\
\text { Finite Automata }\end{array}$ & $\begin{array}{c}\text { Decision } \\
\text { Table }\{\text { extracted } \\
\text { decision table easily } \\
\text { converted into if } \\
\text { then rule, decision } \\
\text { tree, etc\} }\end{array}$ \\
\hline Input Domain & $\begin{array}{c}\text { Categorical, } \\
\text { Continuous, } \\
\text { Discrete, } \\
\text { Ordinal }\end{array}$ & $\begin{array}{c}\text { Capable to handle } \\
\text { all types of data } \\
\text { types }\end{array}$ \\
\hline Accuracy & $\begin{array}{c}\text { Can be } \\
\text { elaborated in } \\
\text { percentage. }\end{array}$ & $\begin{array}{c}\text { High }\{\text { Can be } \\
\text { controlled using } \\
\text { parameter values for } \\
\text { goal settings }\end{array}$ \\
\hline
\end{tabular}

\begin{tabular}{|c|c|c|}
\hline $\begin{array}{c}\text { Comprehensi } \\
\text { bility }\end{array}$ & $\begin{array}{c}\text { Low, Moderate, } \\
\text { High }\end{array}$ & $\begin{array}{c}\text { High (Can be } \\
\text { controlled using } \\
\text { parameter values for } \\
\text { rule length and } \\
\text { number of rules })\end{array}$ \\
\hline Fidelity & $\begin{array}{c}\text { Low, Moderate, } \\
\text { High }\end{array}$ & $\begin{array}{c}\text { High }\{\text { Can be } \\
\text { controlled using } \\
\text { parameter values for } \\
\text { goal settings }\}\end{array}$ \\
\hline Consistency & $\begin{array}{c}\text { Low, Moderate, } \\
\text { High }\end{array}$ & High \\
\hline Translucency & $\begin{array}{c}\text { Pedagogical, } \\
\text { Decompositiona } \\
\text { l, Eclectic }\end{array}$ & Pedagogical \\
\hline Scope & $\begin{array}{c}\text { Classification, } \\
\text { Regression } \\
\text { Yes, No }\end{array}$ & $\begin{array}{c}\text { Classification } \\
\text { Scalable }\end{array}$ \\
\hline Scalability & High & $\begin{array}{c}\text { O(h), Where } \mathrm{h} \text { is } \\
\text { number of features. }\end{array}$ \\
\hline Complexity & $\begin{array}{c}\text { Low, Moderate, } \\
\text { Yes, No }\end{array}$ & Portable \\
\hline Portability & Yesh \\
\hline
\end{tabular}

\section{DIRECTION AND CHALLENGES}

Rule extraction adds power of explanation to underlying model and many researchers has argued for rule extraction due to different reasons. In Tickle et al. [17] has discussed issues related to rule extraction from artificial neural networks and Pennington[32] has try to summarize them. Following are the main research issues in rule extraction area:

1. To handle Accuracy v/s comprehensibility, fidelity v/s performance, and comprehensibility $\mathrm{v} / \mathrm{s}$ fidelity tradeoffs.

2. To achieve most of the goals (Accuracy, fidelity, comprehensibility and performance) of rule extraction techniques simultaneously.

3. To achieve polynomial time complexity not in worst case than atleast for best and average case of algorithm for large scale problems.

4. To overcome the dependency on underlying model.

5. To reduce the dependency on the internal architecture of underlying model like ANN or SVM.

6. To increase performance through eliminating noncontributing features by identifying them from underlying model.

7. To utilize the training data or complete data for training of the underlying model.

8. To check the applicability of an rule extraction algorithm for particular problem domain.

9. To refine the rule using domain specific experience and expert knowledge to bias the learning from a underlying model, so that a more efficient model can be designed and extracted.

10. To use meta learning technique to improve and speedup the rule extraction process.

11. To decide whether rule extraction is for underlying model or from underlying model[23].

12. To analyze the impact of ensemble methods on rule extraction technique and measures for performance evaluation of these kind of methods.

13. To compare multiple models for deciding best amongst them according to the nature of problem.

14. To discriminate whether performance can be increased by training the underlying model like ANN or SVM statically or dynamically.

15. To find the possibilities and effect of training of ANN during the rule extraction process. In general practice 
training of the ANN during the rule extraction process is not allowed.

These issues will direct the researcher to work in the area of rule extraction techniques; solving these issues for example meta learning area in machine learning has tried to identify techniques generally suitable for specific problem characteristics [33]. In Meta learning many interesting and valuable findings have been made, but some areas still remain unexplored [34]. One such area is to examine which types of data sets are suitable for rule extraction and which are not using the characteristics of a data set.

\section{CONCLUSION AND FUTURE SCOPE}

In this paper, we have introduced measure for evaluation and categorization of rule extraction techniques. Our taxonomy allows a clear categorization of rule extraction algorithms. The work available in literature related to taxonomy is limited to ANN whereas proposed taxonomy covers a much broader range of rule extraction techniques. In designing the algorithm KDRuleEx (a recent work in rule extraction area) all best efforts have been made according to KD taxonomy. We have evaluated it using proposed taxonomy and results are favoring our technique. Efforts made in the direction of comparing our work with other researchers and taking most of the associated things together at one place is unique. By considering research issues raised in section 6 , one can develop rule extraction algorithms which can satisfy the future needs of machine intelligence required in the area of prediction.

\section{REFERENCES}

[1] B. Baesens, T. V. Gestel, S. Viaene, M. Stepanova, J. Suykens and J. Vanthienen, "Benchmarking state of the art classification algorithms for credit scoring," vol. 56, no. 6, pp. 627-635, 2003.

[2] U. Johansson, R. Konig and L. Niklasson, "Automatically balancing accuracy and comprehensibility in predictive modeling," 2005.

[3] S. e. a. Thrun, "The MONK's problems: A performance comparison of different learning algorithms," Pittsburgh, pp. 91-197, 1991.

[4] T. Löfström and P. Odqvist, "RULE EXTRACTION IN DATA MINING - FROM A META LEARNING PERSPECTIVE," 2004.

[5] J. Diederich, A. B. Tickle and S. Geva, "Quo Vadis? Reliable and Practical Rule Extraction from Neural Networks," vol. 262, pp. 479-490, 2010.

[6] R. Andrews, J. Diederich and A. Tickle, "Survey and critique of techniques for extracting rules from trained artificial neural networks," Knowledge Based Systems, vol. 8, no. 6, pp. 373-389, 1995.

[7] R. Kohavi, "The Power of Decision Tables," 1995.

[8] J. Huysmans, K. Dejaeger, C. Mues, J. Vanthienen and B. Baesens, "An empirical evaluation of the comprehensibility of decision table, tree and rule based predictive models," vol. 51, no. 1, pp. 141-154, 2011.

[9] J. Vanthienen, "A more general comparison of the decision table and tree: A response," Communications of the ACM, vol. 37, no. 2, pp. 109-113, 1992.
[10] K. K. Sethi, D. K. Mishra and B. Mishra, "KDRuleEx: A Novel Approach for Enhancing User Comprehensibility Using Rule Extraction," in Third International Conference Intelligent Systems, Modelling and Simulation, 2012

[11] M. W. Craven and J. E. Shavlik, "Rule extraction: Where do we go from where?," 1999.

[12] A. Darbari, "Rule Extration from Trained ANN : ASurvey," TU Dresden, 2001

[13] S. K. Ahamed, "Survey of rule extraction methods," ETD Collection for Wayne State University, 2004.

[14] M. G. Augasta and T. Kathirvalavakumar, "Rule extraction from neural networks - A comparative study," in International Conference on , 2012.

[15] J. Huysmans, B. Baesens and J. Vanthienen, "Using rule extraction to improve the comprehensibility of predictive models," Faculty of Economics and Applied Economics, 2006.

[16] W. Duch, R. Setiono and J. M. Zurada, "Computational intelligence methods for rule-based data understanding," 2004.

[17] A. Tickle, R. Andrews, M. Golea and J. Diederich, "The truth will come to light: directions and challenges in extracting the knowledge embedded within mined artificial neural networks," IEEE Transactions on Neural Networks, vol. 9, no. 6, p. 1057-1068, 1998.

[18] G. Towell and J. Shavlik, "The extraction of refined rules from knowledge based neural networks," Machine Learning, vol. 13, no. 1, pp. 71-101, 1993.

[19] M. W. Craven and J. W. Shavlik, "Extracting treestructured representations of trained networks," Advances in Neural Information Processing Systems, vol. 8, pp. 24 30, 1996.

[20] G. P. Schmitz, C. Aldrich and F. S. Gouws, "ANN-DT: An algorithm for extraction of decision trees from artificial neural networks," IEEE Transactions on Neural Networks, vol. 10, no. 6, pp. 1392-1401, 1999.

[21] A. Tickle, M. Orlowski and J. Diederich, "DEDEC: a methodology for extracting rule from trained artificial neural networks," in Workshop on Rule Extraction from Trained Neural Networks, Brighton, UK, 1996.

[22] M. Golea, "On the complexity of rule extraction from neural networks and network querying," in Rule Extraction From Trained Artificial Neural Networks Workshop, Brighton, UK, 1996.

[23] Z. H. Zhou, "Rule extraction: using neural networks or for neural networks?," Journal of Computer Science and vol. 19, no. 2, pp. 249-253, 2004.

[24] P. Domingos, "The Rule of Occam's Razor in Knowledge Discovery," Data Min. Knowl. Discov, vol. 3, no. 4, pp. 409-425, 1999.

[25] U. Johansson, T. Lofstrom, R. Konig, C. Sonstrod and L. Niklasson, "Rule Extraction from Opaque Models- A 
Slightly Different Perspective," 2006.

[26] R. Kohavi and F. Provost, "Glossary of Terms," Special Issue on Applications of Machine Learning and the Knowledge Discovery Process, vol. 30, pp. 271-274, 1998.

[27] H. Jacobsson, "Rule Extraction from Recurrent Neural Networks: A Taxonomy and Review," vol. 17, no. 6, pp. $1-37,2005$

[28] H. Zhang, Y. Zhang and H. Lin, "A comparison study of impervious surfaces estimation using optical and SAR remote sensing images," pp. 148-156, 2012.

[29] R. König, "Predictive Techniques and Methods for Decision Support in Situations with Poor Data Quality," University of Borås. School of Business and Informatics, Sweden, 2009.

[30] C. Mues, J. Huysmans, B. Baesens and J. Vanthienen, "An empirical investigation into the interpretability of data mining models based on decision trees, tables and rules," in 22nd European Conference on Operational Research, Prague, 2007.

[31] A. M. Moreno Garcia, M. Verhelle and J. Vanthienen, "An overview of decision table literature 1982-2000," in organized by the Research Group on AI in Accounting , Huelva, Spain, 2000.
[32] M. Pennington, "C4.5 Rule Preceded by an Artificial Neural Network Ensemble for Medical Diagnosis," 2003.

[33] A. Kalousis, J. Gama and M. Hilario, "On Data and Algorithms: Understanding Inductive Performance," Special Issue on Meta-Learning, vol. 54, no. 3, pp. 275 312, 2004.

[34] T. Löfström and U. Johansson, "Predicting the Benefit of Rule Extraction - A Novel Component in Data Mining," vol. 7, no. 3, pp. 78-108, 2005.

[35] N. Barakat and J. Diederich, "Eclectic rule-extraction from support vector machines," vol. 2(1), pp. 59-62, 2005.

[36] R. KÄonig and L. Niklasson, "Automatically balancing accuracy and comprehensibility in predictive modeling," 2005.

[37] U. Johansson, R. König and L. Niklasson, "The Truth is in There: Rule Extraction from Opaque Models Using Genetic Programming," in 17th Florida Artificial Intelligence Research Symposium (FLAIRS), Miami, FL, 2004. 